\title{
Sparsity Enhanced Beamforming in The Presence of Coherent Signals
}

\author{
Bao Gen Xu', Yi He Wan ${ }^{1}$, Qun Wan ${ }^{2}$, Si Long Tang ${ }^{1}$, Xue Ke Ding ${ }^{1}$, Lin Zou ${ }^{2}$ \\ ${ }^{1}$ Joint Lab of Array Signal Processing, TongFang Electronic Science and Technology \\ Co. Ltd., Jiujiang, China, 332007 \\ ${ }^{2}$ Department of Electronic Engineering, University of Electronic Science and Technolo- \\ gy of China, Chengdu, China, 611731
}

\begin{abstract}
In order to find the directions of coherent signals, a sparsity enhanced beamforming method is proposed. The minimum variance in the proposed method corresponds to the orthogonal relationship between the noise subspace and the sparse representation of the received signal vector, whereas the distortless response corresponds to the non-orthogonal relationship between the signal subspace and the sparse representation of the received signal vector. The proposed sparsity enhanced method is carried out by the iterative reweighted Lp-norm constraint minimization for direction finding of coherent signals. Simulation results are provided to show that it has better performance than the existing algorithms in presence of coherent signals.
\end{abstract}

Keywords: distortless response of signal subspace; minimum variance of noise subspace; sparse representation; direction finding; coherent signals

\section{Introduction}

Source localization has been of interest in the past few decades and played a fundamental role in many applications involving electromagnetic, acoustic, biomedical, seismic sensing, etc. An important goal for source localization methods is to be able to locate coherent signals in the presence of multipath propagation [1]. Many advanced methods for the localization of incoherent signals attain super-resolution by exploiting the separability of a small number of signals. The most well-known existing methods include Capon's method [2], beam-forming [3] and its relevant algorithms [4], and subspace based methods such as MUSIC [5]. However, most of them could not deal with the coherent signals.

Recently, the usage of sparse feature of signals has evolved very rapidly, finding applications in several kinds of signal processing problems. There has also been some emerging research of these ideas in the context of spatial spectrum estimation, beam-forming, and direction finding by antenna array $[3,4]$. Sacchi et al. took advantage of Cauchy-prior to introduce sparsity in spectrum estimation and solved the nonlinear optimization problem by iterative approaches [6]. Jeffs made use of an Lp-norm penalty with $0 \leq \mathrm{p} \leq 1$ to enforce sparse feature deduced from several applications, including sparse antenna array design [7]. Gorodnitsky and Rao used a recursive weighted minimum-norm algorithm called focal under-determined system solver (FOCUSS) to make use of sparsity of spatial spectrum in the problem of DOA estimation [8]. The work of Fuchs [9] was also involved in sparse signal localization under the assumption that the number of snapshots is abundant. In [3], 
In order to improve the beam-pattern, a total variation minimisation of the whole beam pattern is incorporated to encourage large array gains accumulated in the mainlobe and small trivial array gains gathered in the side-lobes, while revising the sparse constraint only on the sidelobe.

All these methods are based on the sparse representation of the received vector of the array as a sparse linear combination of direction vectors. The L1 penalty for sparsity and the L2 penalty for noise are often ustilized to recover the sparse signal representation. To mitigate the effect of measurement noise and reduce the calculation, a novel DOA estimation method, L1-SVD [10], was proposed, which sparsely represented the signal subspace by an overcomplete basis and assumed that DOAs of incoming signals are usually very sparse relative to the whole spatial domain. It is carried out by L1-norm constraint minimization due to it is a convex problem. However, L1-norm constraint minimization has a drawback that larger coefficients of signal are punished more heavily than smaller coefficients, unlike the more impartial punishment of the L0-norm constraint minimization [11]. This causes the degradation of sparse signal recovery performance based on regular L1-norm constraint minimization.

In this paper, we focus on the problem of direction finding for coherent signals. The methodology of the iterative reweighted Lp-norm constraint minimization is expanded from the array data to signal and noise subspace for direction finding of coherent signals. Making use of the orthogonality between noise subspace and sparse representation of received signal vector, the objective of Lpnorm constrained minimization variance distortless response (MVDR) can be achieved.

\section{Problem formulation}

First use the equation editor to create the equation. Then select the "Equation" markup style.

Consider a uniform circular array (UCA) that consists of $M$ antenna. The radius of the circle is $r$. Assume that the sources $s_{k}(t)$ come from azimuth $\theta_{k}$ in the far field of the array, $k=1,2, \cdots, K$, $K$ is the number of sources. The received signal vector of UCA can be expressed as:

$$
\mathbf{x}(t)=\sum_{k=1}^{K} \mathbf{a}\left(\theta_{k}\right) s_{k}(t)+\mathbf{v}(t)
$$

where $\mathbf{x}(t)$ is the received signal vector, $t$ is sampling moments, $\mathbf{v}(t)$ is the receiver noise vector, $\mathbf{a}\left(\theta_{k}\right)$ is direction vector corresponding to azimuth $\theta_{k}$. The $m$-th component of $\mathbf{a}\left(\theta_{k}\right)$ is

$$
\begin{gathered}
\mathbf{a}_{m}\left(\theta_{k}\right)=e^{j 2 \pi f_{c} r \cos \left(\theta_{k}-(m-1) \frac{2 \pi}{M}\right)}, \\
m=1,2, \cdots, M
\end{gathered}
$$

When there are coherent signals, i.e., $s_{k}(t)=\alpha_{k i} s_{i}(t)$ for constant $\alpha_{k i}$ and $1 \leq k \neq i \leq K$, we only have $L$ different and incoherent signals where $L<K$.

The sample autocorrelation matrix of the received vector $\mathbf{x}\left(t, \theta_{k}\right)$ is:

$$
\mathbf{R}=\frac{1}{T} \sum_{t=1}^{T} \mathbf{x}(t) \mathbf{x}^{H}(t)
$$

where $T$ represents the number of received signal vectors of UCA, and []$^{H}$ complex conjugate transposition. The singular value decomposition of the sample autocorrelation matrix is

$$
\mathbf{R}=\mathbf{U} \boldsymbol{\Lambda} \mathbf{U}^{H}
$$

where $\boldsymbol{\Lambda}$ is a diagonal matrix whose diagonal elements correspond to the singular value of $\mathbf{R}, \mathbf{U}$ is matrix whose column vectors are singular vectors of $\mathbf{R}$, 
$\mathbf{u}_{1}, \mathbf{u}_{2}, \mathbf{u}_{3}, \cdots, \mathbf{u}_{M}$, corresponding to the singular values, $\lambda_{1} \geq \lambda_{2}>\lambda_{3} \geq \cdots \geq \lambda_{M}$.

According to the subspace decomposition approach, the noise subspace of the sample autocorrelation matrix is:

$$
\mathbf{Q}_{n}=\left[\begin{array}{llll}
\mathbf{u}_{L+1} & \mathbf{u}_{L+1} & \cdots & \mathbf{u}_{M}
\end{array}\right]
$$

where $L$ is the number of incoherent signals. The problem is to estimate the azimuth $\theta_{k}, k=1,2, \cdots, K$, for all the signals whatever they are coherent or incoherent signals.

\section{Sparsity enhanced MVDR}

According to the criterion of minimum variance and distortless response (MVDR), we have the spatial spectrum:

$$
\begin{gathered}
\mathbf{f}_{\text {mvdr }}\left(\varphi_{n}\right)=\min _{\mathbf{w}} \mathbf{w}^{H} \mathbf{R} \mathbf{w} \\
\text { s.t. } \quad \mathbf{a}^{H}\left(\varphi_{n}\right) \mathbf{w}=1
\end{gathered}
$$

where the weighting vector $\mathbf{w} \in C^{M}$ and $\varphi_{n}$ is the searching grid of azimuth, e.g., $\varphi_{n}=0,1, \ldots, 359$ degree, $n=1,2, \ldots, N$ and $N=360$.

Because the above problem is a quadratic optimization with linear constraint, it is easy to calculate the spatial spectrum in the closed-form as given by

$$
\mathbf{f}_{\mathrm{mvdr}}\left(\varphi_{n}\right)=\frac{1}{\mathbf{a}^{H}\left(\varphi_{n}\right) \mathbf{R}^{-1} \mathbf{a}\left(\varphi_{n}\right)}
$$

However, it is usually not sparse enough in the spatial domain.

To take the advantage of sparse property of the spatial spectrum, we enhance the criterion of minimum variance and distortless response (MVDR) by sparsity constraint. The problem of sparsity enhanced MVDR can be described as:

$$
\begin{gathered}
\mathbf{f}_{\text {semvdr }}=\arg \min _{\mathbf{w}}\left\|\mathbf{Q}_{n} \mathbf{A} \mathbf{w}\right\|_{F}+\beta\|\mathbf{w}\|_{0} \\
\text { s.t. } \quad \mathbf{u}_{1}{ }^{H} \mathbf{A} \mathbf{w}=1
\end{gathered}
$$

where $\|\mathbf{w}\|_{0}$ is the number of non-zero components of the weighting vector $\mathbf{w} \in C^{N}$,

$$
\mathbf{A}=\left[\begin{array}{llll}
\mathbf{a}\left(\varphi_{1}\right) & \mathbf{a}\left(\varphi_{2}\right) & \cdots & \mathbf{a}\left(\varphi_{N}\right)
\end{array}\right]
$$

Unfortunately, the above problem of nonlinear optimization is an NP-hard problem. A remedy is to use the Lp-norm constraint minimization instead and solve it with iterative adaptive algorithm.

$$
\begin{gathered}
\mathbf{f}_{\text {semvdr }}=\arg \min _{\mathbf{w}}\left\|\mathbf{Q}_{n} \mathbf{A} \mathbf{w}\right\|_{F}+\beta\|\mathbf{G w}\|_{F} \\
\text { s.t. } \quad \mathbf{u}_{1}^{H} \mathbf{A} \mathbf{w}=1
\end{gathered}
$$

where

$$
\mathbf{G}=\operatorname{diag}\left(|\mathbf{w}|^{p / 2-1}\right)
$$

It should be worth noting that $\|\mathbf{G w}\|_{F}=\|\mathbf{w}\|_{p}$. To avoid the drawback that larger coefficients of the weighting vector $\mathbf{w}$ are punished more heavily than smaller coefficients, unlike the more impartial punishment of $\|\mathbf{w}\|_{0}$ constraint minimization, we often choose $0 \leq p \leq 0.5$.

The optimization problem (9) is nonconvex and can be rewritten as

$$
\mathbf{f}_{\text {semvdr }}=\arg \min _{\mathbf{w}} \mathbf{w}^{H}\left(\mathbf{A}^{H} \mathbf{Q}_{n}^{H} \mathbf{Q}_{n} \mathbf{A}+\beta \mathbf{G}^{2}\right) \mathbf{w}
$$

$$
\text { s.t. } \mathbf{u}_{1}^{H} \mathbf{A w}=1
$$

However, if we assume that $\mathbf{G}$ is independent with the weighting vector $\mathbf{w}$, it is easy to solve the above problem in closed-form as

$$
\mathbf{w}=\frac{1}{\mathbf{u}_{1}^{H} \mathbf{A} \mathbf{B} \mathbf{A}^{H} \mathbf{u}_{1}} \mathbf{B} \mathbf{A}^{H} \mathbf{u}_{1}
$$

where

$$
\mathbf{B}=\left(\mathbf{A}^{H} \mathbf{Q}_{n}^{H} \mathbf{Q}_{n} \mathbf{A}+\beta \mathbf{G}^{2}\right)^{-1}
$$

Therefore, we can summarize the iterative adaptive algorithm to solve the problem (11) as the following.

(1) Initialization: $\mathbf{w}_{n}=\mathbf{f}_{\text {mvdr }}\left(\varphi_{n}\right)$;

(2) Update: $\mathbf{G}=\operatorname{diag}\left(|\mathbf{w}|^{p / 2-1}\right)$ and $\mathbf{B}=\left(\mathbf{A}^{H} \mathbf{Q}_{n}^{H} \mathbf{Q}_{n} \mathbf{A}+\beta \mathbf{G}^{2}\right)^{-1} ;$

(3) Upate: $\mathbf{w}=\frac{1}{\mathbf{u}_{1}^{H} \mathbf{A B} \mathbf{A}^{H} \mathbf{u}_{1}} \mathbf{B} \mathbf{A}^{H} \mathbf{u}_{1}$; 
(4) Repeat (Step 2) and (Step 3), until the difference between $\mathbf{w}$ obtained by the adjacent steps is small enough.

Finally, the spatial spectrum of the sparsity enhanced MVDR is given by

$$
\mathbf{f}_{\text {semvdr }}=\mathbf{w}
$$

Obviously, there are many differences between MVDR and SEMVDR. First, the dimension of the weighting vector of MVDR is the same as the number of antenna of UCA, whereas weighting vector of SEMVDR is the same as the number of the searching grid of azimuth. Second, the spatial spectrum of MVDR is a quadratic function of the weighting vector, whereas the spatial spectrum of SEMVDR is directly the weighting vector. Finally, the spatial spectrum of SEMVDR is explicitly sparse, whereas MVDR takes no advantage of sparsity of the spatial spectrum.

Because the above SEMVDR algorithm only find the sparse solution, there may be some signals that could not be found by the peak position of $\mathbf{f}_{\text {semvdr }}$. When the directions of signals are estimated from the peak position of $\mathbf{f}_{\text {semvdr }}$, we should modify the signal subspace and noise subspace as

and

$$
\mathbf{u}_{1}=\mathbf{P}^{\perp} \mathbf{u}_{1}
$$

$$
\mathbf{Q}_{n}=\mathbf{P}^{\perp} \mathbf{Q}_{n}
$$

where $\mathbf{P}^{\perp}$ is the orthogonal projection matrix of matrix whose column vectors are the direction vectors corresponding to the the directions of signals estimated from the peak position of $\mathbf{f}_{\text {semvdr }}$. Then, we should repeat the above SEMVDR algorithm until no significant peak is found in $\mathbf{f}_{\text {semvdr }}$.

\section{Simulation Result}

We consider a uniform circular array of $M=9$ antenna with radius $r=40$ meters. The wavelength of the narrowband signals is 15 meters. Three zero-mean narrowband signals in the far-field impinge upon this array from distinct directions of arrival (DOA), i.e., 50.3, 78.5 and 112.4 degree. The first and third signal are coherent. The total number of snapshots is $\mathrm{T}=64$, the signal to noise ratio (SNR) is $9 \mathrm{~dB}$, and $\beta=0.1^{6}$. In Figs 1 and 2, we compare the spatial spectrum obtained using our proposed method with those of MVDR and MUSIC methods.

In Fig.1, we can see that MVDR and MUSIC only detect the second signal whose direction vector is orthogonal to the noise subspace, and are unable to detect the coherent signals whose direction vectors are not orthogonal to the noise subspace. On the contrary, in Fig.2, we can see that MVDR and MUSIC only detect the second signal whose direction vector is orthogonal to the noise subspace, and are unable to detect the coherent signals whose direction vectors are not orthogonal to the noise subspace.

\section{Conclusion}

The novelty and advantage of our technique is that it is able to find the directions of coherent signals. Though the proposed sparsity enhanced method is carried out by the iterative reweighted Lp-norm constraint minimization, the number of iteration is usually not more than 20. Simulation results show that it has better performance than the existing algorithms in the presence of coherent signals.

\section{Acknowledgment}

This work was supported by the National Natural Science Foundation of China under grant 61172140 and "Excellent Teaching Team Support Project (Graduate student part) " of University of Electronic Science and Technology of 
China (No. A1098522-02) and joint laboratory project of UESTC-713 shortwave \& ultra-short-wave array signal processing.
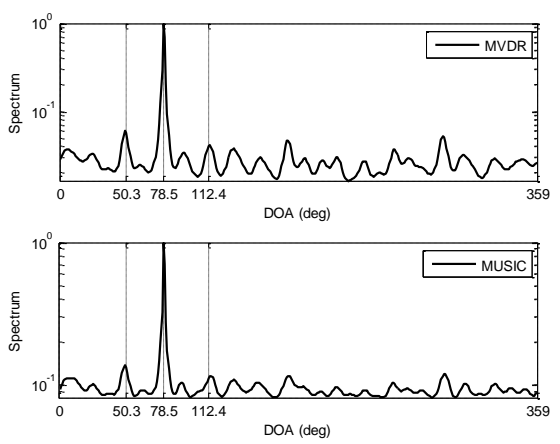

Fig. 1. Spatial spectrum of MVDR and MUSIc.
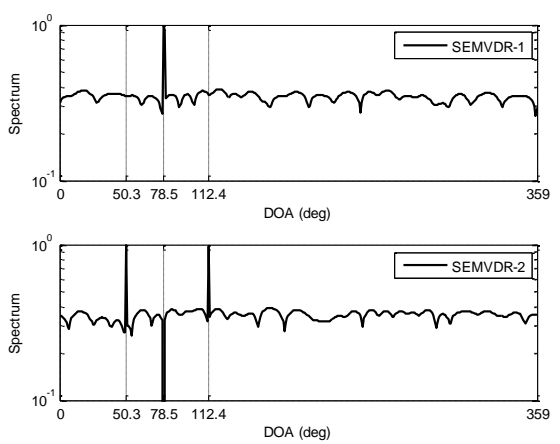

Fig. 2. Spatial spectrum of two-step's sparsity enhanced MVDR (SEMVDR-1 and SEMVDR-2).

\section{References}

[1] Q. Wan, B. G. Xu, J. H. Yin, F. Fang, Y. H. Wan, S. L. Tang, "An approach to manifold estimation for antenna array in situation of interferences," IET International Radar Conference 2013, Xi An, China, Apr. 14-16, 2013

[2] J. Capon, "High resolution frequency-wavenumber spectrum analysis," Proc. IEEE, vol. 57, no. 8, pp.14081418, Aug. 1969.
[3] Y.P. Liu, Q. Wan, " Robust beamformer based on total variation minimisation and sparse constraint," Electronic Letters, vol.46, no.25, pp.16971699, 2010.

[4] Y. Zhang, B.P. Ng and Q. Wan, Sidelobe suppression for adaptive beamforming with sparse constraint on beam pattern, Electronics Letters, vol.44, no.10, pp.615-616, 2009.

[5] R. O. Schmidt, "Multiple emitter location and signal parameter estimation," IEEE Trans. Antennas Propag., vol. 34, no. 3, pp.276-280, Mar. 1986.

[6] M. D. Sacchi, T. J. Ulrych, and C. J. Walker, "Interpolation and extrapolation using a high-resolution discrete fourier transform," IEEE Trans. Signal Process., vol. 46, no. 1, pp.31-38, Jan. 1998.

[7] B. D. Jeffs, "Sparse inverse solution methods for signal and image processing applications," Proc. IEEE Int. Conf. Acoust., Speech, Signal Process., vol. 3, pp.1885-1888, 1998.

[8] I. F. Gorodnitsky, and B. D. Rao, "Sparse signal reconstruction from limited data using FOCUSS: A reweighted minimum norm algorithm," IEEE Trans. Signal Process., vol. 45, no. 3, pp.600-616, Mar. 1997.

[9] J. J. Fuchs, "On the application of the global matched filter to DOA estimation with uniform circular arrays," IEEE Trans. Signal Process., Vol. 49, No. 4, pp.702-709, Apr. 2001.

[10] D. M. Malioutov, M. Cetin, and A. S. Willsky, "A sparse signal reconstruc tion perspective for source localiza tion with sensor arrays," IEEE Trans. Signal Process., vol. 53, no. 8, pp.3010-3022, Aug. 2005.

[11]E. J. Cands, M. B. Wakin, and S. P. Boyd, "Enhancing sparsity by re weighted L1 minimization," Journal of Fourier Analysis and Applications, vol. 14, no. 5, pp.877-905, Dec. 2008. 